\title{
Numerical Study of Flapping-Wing Flow Physics in Nonuniform Freestream Using the High-Order Spectral Difference Method on Dynamic Unstructured Grids
}

\author{
Naresh Poudel ${ }^{1}$ and Meilin $\mathrm{Yu}^{2}$ \\ University of Maryland Baltimore County (UMBC), Baltimore, MD 21250
}

\begin{abstract}
In this paper, a numerical study is conducted to understand the impact of nonuniform freestream on the aerodynamic performance of a flapping foil. Specifically, an unsteady flow environment is generated by stationary inline or staggered circular cylinder arrays upstream. A 2D NACA0012 airfoil is then placed at different locations downstream of the cylinder arrays, and oscillates in the nonuniform freestream. Unsteady flows over the flapping foil are numerically simulated by solving the 2D low-Mach-number-preconditioned compressible Naiver-Stokes equations on deformable unstructured grids using the high-order spectral difference method. The flow fields over a pitching airfoil in unsteady flow environments and the corresponding aerodynamic forces are analyzed and compared under different flow conditions. It is observed that the nonuniform freestream can significantly affect the unsteady vortex dynamics of a flapping foil, thus modifying the thrust and lift generation.
\end{abstract}

\section{Nomenclature}

$\begin{array}{ll}A & =\text { pitching amplitude } \\ d & =\text { cylinder diameter } \\ C_{p} & =\text { pressure coefficient } \\ C_{L} & =\text { lift coefficient per unit span } \\ C_{L, R M S} & =\text { root mean square of lift coefficient per unit span } \\ C_{T} & =\text { thrust coefficient per unit span } \\ C_{T, R M S} & =\text { root mean square of thrust coefficient per unit span } \\ R e & =\text { Reynolds Number } \\ \mathrm{c} & =\text { chord } \\ U_{\infty} & =\text { freestream velocity } \\ \theta_{m} & =\text { mean angle of attack } \\ \theta_{0} & =\text { amplitude of the pitching angle } \\ f & =\text { oscillation frequency } \\ S_{t} & =\text { Strouhal Number } \\ k & =\text { reduced frequency } \\ \mathrm{T} & =\text { time } \\ \Delta t & =\text { time step } \\ \varnothing & =\text { phase difference }\end{array}$

\footnotetext{
${ }^{1}$ Graduate Student, Department of Mechanical Engineering, npoudel1@umbc.edu, and AIAA Student Member.

${ }^{2}$ Assistant Professor, Department of Mechanical Engineering, mlyu@umbc.edu, and Senior AIAA Member.
} 


\section{Introduction}

The increasing use of micro air vehicles (MAVs) for civilian and military missions has attracted extensive attention of researchers to understand the unsteady flapping wing aerodynamics. ${ }^{1}$ MAVs usually work under nonuniform flow conditions and the nonuniformity in flow arises due to the low atmospheric urban areas and gusty wind conditions. Nonuniform flow conditions can cause flight stability and control problems for MAVs. ${ }^{2,3}$ Due to the small mass and low speed of the MAVs, the impact of gusty wind and unsteady flow environment on their flight performance is significant. ${ }^{3}$ It has been a challenge for designers to design MAVs to overcome the flight instability caused by gusty conditions. ${ }^{4}$ However, most studies for the flapping motion of the birds and insects have neglected the effect of wind gust on the stability of the motion. Hence the study of aerodynamics performance of flapping wings under the impacts of gusty conditions and sudden change of flight conditions is considered as an important aspect for the better design of promising MAVs.

Many studies have been performed to study the flight instabilities on MAVs under gusty conditions, ground effects and sudden change in flight condition. Objects in tandem configurations with flapping wings placed downstream are usually used in these studies, and sometimes, the gust frequencies are used to mimic the gust condition. Shyy et al. ${ }^{5}$ performed the experimental and numerical study on the rigid and flexible airfoils under the effects of gust, and demostrated that the flexible airfoil performs better under gust conditions than rigid airfoil in terms of lift to drag ratio. Wang and Huang ${ }^{6}$ studied numerically the 2D flapping wing aerodynamics, and reported that the wind gust can contribute negatively to lift and thrust, causing flight insatbility. Prater and $\operatorname{Lian}^{7}$ numerically studied the flight characteristics of stationary and flapping single and tandem wing configuration, under uniform flow with sinusoidal velocity inflow conditions as a model of gust. Lian and Shyy ${ }^{8}$ found that gusty flow could produce the thrust during deaccelerating stage. Jones and Yamaleev ${ }^{9}$ numerically studied the performance of flapping wings under the influence of different gust conditions, namely, frontal, downward and side gust, and demostrated that the flapping wings can effectively recover form gusty wind fluctuations. $\operatorname{Lian}^{3}$ conducted the numerical study of flapping wings and reported that the gust fluctuations effects can be alleviated under different flapping kinematics. Gao and $\mathrm{Lu}^{10}$ reported three differrent behaviours of force, namely, force enhancement, force reduction, and force recovery, closely associated with the evolution of vortex structures due to the ground effects in insect normal hovering.

Chen and Choa ${ }^{11}$ performed the experimental study on the effect of turbulent wakes on a pitching airfoil. In this work, a small thin cylinder was placed upstream at different vertical positions relative to the pitching airfoil at Reynolds number 80,000 with low reduced frequencies range of 0.01-0.04. They compared the aerodynamic forces and moments with those from the undistrubed freestream case, and found that the dynamic stall occurs at larger angle of attacks due to the turbulent wake, and the growth of the leading-edge suction peak is sensitive to the vertical position of the cylinder. Wang et al. ${ }^{12}$ investigated the effects of turbulence intensity on the aerodynamic forces of NACA0012 at low Reynolds numbers experimentally. They concluded that the flow separation point shifts upstream with increasing Reynolds numbers but downstream with increasing turbulence intensity. Gharali and Johnson ${ }^{13}$ conducted a 2D numerical study of a pitching airfoil with the nonuniform inflow velocity, showing the contribution of unsteady incident velocity on the vortical structure and resultant aerodynamic loads. Merrill and Peet ${ }^{14}$ performed numerical study on a pitching airfoil at the Reynolds number 44,000 and reduced frequency 0.16 in the presence of a turbulent wake produced by an upstream small cylinder. Their study showed the significant impacts of the upstream wake on forces and moments acting on the airfoil. They also found that the dynamic stall starts at a later time at high angle of attacks due to delayed formation and detachment of the dynamic stall vortex. Several other previous researchers ${ }^{15-21}$ have demostrated that the impact of unsteady inflow on aerodynamic loads is also applicable to helicopters and maneuverable wings working in the environment with freestream velocity fluctuations.

In the current work, the aim is to investigate the aerodynamics performance of flapping wings in a highly unsteady flow environment, which is generated by circular cylinders arranged in an inline or staggered configuration upstream. As is known, the bioinspired flows such as those similar to the flows 
induced by the flapping motion of birds, insects and aquatic animals are usually vortex-dominated. Highorder computational fluid dynamics (CFD) methods had been proven more accurate and efficient than the convectional first- and second-order methods to capture the complex vortex-dominated flow structures. In this study, a high-order spectral difference (SD) method with dynamic mesh deformation ${ }^{22}$ is used to simulate the unsteady flapping-wing aerodynamics in nonuniform freestream.

The remainder of the paper is structured as follows. In section II, a brief introduction of the high-order spectral difference method is discussed. A problem statement for the numerical simulation is mentioned in Section III. In Section IV, the results on the flapping-wing aerodynamics under nonuniform flow conditions are presented and discussed. Section V briefly concludes the work and discusses the future work plan.

\section{Numerical Methods}

\section{II.1. Governing Equations}

Unsteady compressible Navier-Stokes equations in conservative form is considered in the physical domain $(t, x, y, z)$ as follows:

$$
\frac{\partial Q}{\partial t}+\frac{\partial F}{\partial x}+\frac{\partial G}{\partial y}+\frac{\partial H}{\partial z}=0
$$

where $Q=(\rho, \rho u, \rho v, \rho w, E)^{T}$ are conservative variables, $\rho$ is the density of fluid, $, u, v$ and $w$ are the $\mathrm{x}$, $\mathrm{y}$ and $\mathrm{z}$ components of velocity and $E$ is the total energy given by $E=\frac{p}{\gamma-1}+\frac{1}{2} \rho\left(u^{2}+v^{2}+w^{2}\right)$ for a perfect gas law in which $p$ is the pressure and $\gamma$ is the constant specific heat capacity ratio. The total energy equation closes the solution system. $F, G$ and $H$ are total flux vectors including the inviscid and viscous flux terms, which are expressed as

$$
\begin{aligned}
& F=F^{i}-F^{v}=\left\{\begin{array}{c}
\rho u \\
\rho u^{2}+p-\tau_{x x} \\
\rho v u-\tau_{x y} \\
\rho w u-\tau_{x z} \\
u(E+p)-u \tau_{x x}-v \tau_{x y}-w \tau_{x z}-\frac{\mu C_{p}}{P r} T_{x} \\
\rho v
\end{array}\right\} \\
& G=G^{i}-G^{v}=\left\{\begin{array}{c}
\rho v \\
\rho u v-\tau_{y x} \\
\rho v^{2}+p-\tau_{y y} \\
\rho w v-\tau_{y z} \\
v(E+p)-u \tau_{y x}-v \tau_{y y}-w \tau_{y z}-\frac{\mu C_{p}}{P r} T_{y}
\end{array}\right\} \\
& H=H^{i}-H^{v}=\left\{\begin{array}{c}
\rho r \\
\rho u w-\tau_{z x} \\
\rho v w-\tau_{z y} \\
\rho w^{2}+p-\tau_{z z} \\
w(E+p)-u \tau_{z x}-v \tau_{z y}-w \tau_{z z}-\frac{\mu C_{p}}{P r} T_{z}
\end{array}\right\} \text {, }
\end{aligned}
$$

where $\mu$ is the dynamic viscosity, $C_{p}$ is the specific heat at constant pressure, $\operatorname{Pr}$ is the Prandtl number and $T$ is the temperature. For the Newtonian fluids, the viscous stresses are given as follows:

$$
\tau_{x x}=2 \mu\left(u_{x}-\frac{u_{x}+v_{y}+w_{z}}{3}\right), \quad \tau_{y y}=2 \mu\left(v_{y}-\frac{u_{x}+v_{y}+w_{z}}{3}\right),
$$




$$
\begin{array}{cc}
\tau_{z z}=2 \mu\left(w_{z}-\frac{u_{x}+v_{y}+w_{z}}{3}\right), & \tau_{x y}=\tau_{y x}=\mu\left(v_{x}+u_{y}\right), \\
\tau_{x z}=\tau_{z x}=\mu\left(w_{x}+u_{z}\right), & \tau_{y z}=\tau_{z y}=\mu\left(w_{y}+v_{z}\right) .
\end{array}
$$

The governing equation (1) in the physical domain $(t, x, y, z)$ is transformed to computational domain $(\tau, \xi, \eta, \zeta)$ as shown in Eq. (4). In the coordinate transformation, $\tau=t$ and $(\xi, \eta, \zeta) \in(-1,1) \times(-1,1) \times$ $(-1,1)$ is standard cubic element in the computational domain.

$$
\frac{\partial \widetilde{Q}}{\partial \tau}+\frac{\partial \widetilde{F}}{\partial \xi}+\frac{\partial \widetilde{G}}{\partial \eta}+\frac{\partial \widetilde{H}}{\partial \zeta}=0
$$

where

$$
\left\{\begin{array}{c}
\widetilde{Q}=|J| Q \\
\widetilde{F}=|J|\left(Q \xi_{\tau}+F \xi_{x}+G \xi_{y}+H \xi_{z}\right) \\
\widetilde{G}=|J|\left(Q \eta_{\tau}+F \eta_{x}+G \eta_{y}+H \eta_{z}\right) \\
\widetilde{H}=|J|\left(Q \zeta_{\tau}+F \zeta_{x}+G \zeta_{y}+H \zeta_{z}\right)
\end{array} .\right.
$$

During the coordinate transformation, the Jacobian matrix can be written as the following form:

$$
J=\frac{\partial(x, y, z, t)}{\partial(\xi, \eta, \zeta, \tau)}=\left(\begin{array}{cccc}
x_{\xi} & x_{\eta} & x_{\zeta} & x_{\tau} \\
y_{\xi} & y_{\eta} & y_{\zeta} & y_{\tau} \\
z_{\xi} & z_{\eta} & z_{\zeta} & z_{\tau} \\
0 & 0 & 0 & 1
\end{array}\right) .
$$

The inverse transformation must also exist for a non-singularity transformation, which can be related to the Jacobian matrix as Eq. (7):

$$
J^{-1}=\frac{\partial(\xi, \eta, \zeta, \tau)}{\partial(x, y, z, t)}=\left(\begin{array}{cccc}
\xi_{x} & \xi_{y} & \xi_{z} & \xi_{t} \\
\eta_{x} & \eta_{y} & \eta_{z} & \eta_{t} \\
\zeta_{x} & \zeta_{y} & \zeta_{z} & \zeta_{t} \\
0 & 0 & 0 & 1
\end{array}\right)
$$

\section{II.2. Space Discretization and Time Integration Methods}

A 3D SD method on dynamic unstructured grids developed in the Ref. 22 is used to solve the governing equations. For completeness, the SD formulation in a standard hexahedral element is expressed as

$$
\begin{aligned}
\frac{\partial \widetilde{Q}(\xi, \eta, \zeta)}{\partial \tau} & +\sum_{i=1}^{N+1} \sum_{j=1}^{N} \sum_{k=1}^{N}\left(\tilde{F}_{i, j, k}^{i n v, I}-\widetilde{F}_{i, j, k}^{v i s, I}\right) \cdot \frac{d}{d \xi} l_{F P s, i}(\xi) \cdot l_{S P S, j}(\eta) \cdot l_{S P S, k}(\zeta) \\
& +\sum_{i=1}^{N} \sum_{j=1}^{N+1} \sum_{k=1}^{N}\left(\widetilde{G}_{i, j, k}^{i n v, I}-\tilde{G}_{i, j, k}^{v i s, I}\right) \cdot l_{S P s, i}(\xi) \cdot \frac{d}{d \eta} l_{F P s, j}(\eta) \cdot l_{S P s, k}(\zeta) \\
& +\sum_{i=1}^{N} \sum_{j=1}^{N} \sum_{k=1}^{N+1}\left(\widetilde{H}_{i, j, k}^{i n v, I}-\widetilde{G}_{i, j, k}^{v i s, I}\right) \cdot l_{S P s, i}(\xi) \cdot l_{S P s, j}(\eta) \cdot \frac{d}{d \zeta} l_{F P s, k}(\zeta)=0 .
\end{aligned}
$$

Note that two sets of points, namely solution points and flux points, are used in the SD method. In Eq. (8), $l_{F P S}$ stands for the flux-points-based Lagrange polynomial, $l_{S P S}$ stands for the solution-points-based 
Lagrange polynomial, and the superscript ' $I$ ' indicates that the fluxes are continuous across different elements. To ensure the flux continuity on element surfaces, the common inviscid fluxes are reconstructed with the $\mathrm{AUSM}^{+}$-up Riemann solver for all speeds ${ }^{24}$, and the common viscous fluxes are reconstructed with the 'BR1' approach developed in the Ref. 25.

The explicit three-stage strong stability preserving (SSP) Runge-Kutta method ${ }^{26}$ is used for time integration. For any ordinary differential equation in the form $d Q / d t=R(Q)$, the three-stage SSP Runge-Kutta method is given by

$$
\left\{\begin{array}{c}
Q^{(1)}=Q^{n}+\Delta t R\left(Q^{n}\right) \\
Q^{(2)}=\frac{3}{4} Q^{n}+\frac{1}{4} Q^{(1)}+\frac{1}{4} \Delta t R\left(Q^{(1)}\right) \\
Q^{n+1}=\frac{1}{3} Q^{n}+\frac{2}{3} Q^{(2)}+\frac{2}{3} \Delta t R\left(Q^{(2)}\right)
\end{array}\right.
$$

\section{II.3. Dynamic Grids Deformation}

In the current work, the moving grid deformation algorithm is obtained from the earlier work ${ }^{22,23}$ which used the blending function approach to reconstruct the whole physical domain. The fifth order blending function with $r(0)=0$ and $r(1)=1$ is used for dynamic mesh deformation. It is written as

$$
r_{5}(s)=10 s^{3}-15 s^{4}+6 s^{5}, \quad s \in[0,1]
$$

where $\mathrm{s}$ represents the normalized distance between present mesh node and the moving boundaries. If $r(0)=0$, the mesh node will move with the moving boundary; but if $r(1)=1$, the mesh node will not move. The change in the position vector $\vec{P}$ for an arbitrary mesh node can be obtained as follows:

$$
\Delta \vec{P}_{\text {present }}=\left(1-r_{5}\right) \Delta \vec{P}_{\text {rigid }}
$$

The new mesh nodes after deformation are calculated by adding $\Delta \vec{P}$ to $\vec{P}$.

\section{Problem Statement}

In this study, the NACA0012 airfoil is undergoing a pitching motion, which is expressed as:

$$
\theta(t)=\theta_{m}+\theta_{0} \sin (2 \pi f t+\emptyset)
$$

where $\theta_{m}$ is the mean angle of attack, $\theta_{0}$ is the amplitude of the pitching angle, $\varnothing$ is the initial phase, $f$ is the oscillating frequency and $t$ is the dimensional time. The reduced frequency, $\mathrm{k}$ and Strouhal number, $S_{t}$ are given as

$$
\begin{aligned}
k & =\frac{\pi f c}{U_{\infty}}, \\
S_{t} & =\frac{2 f A}{U_{\infty}},
\end{aligned}
$$

where $c$ is the chord length of airfoil, $A$ is the pitching amplitude and $U_{\infty}$ is the freestream velocity.

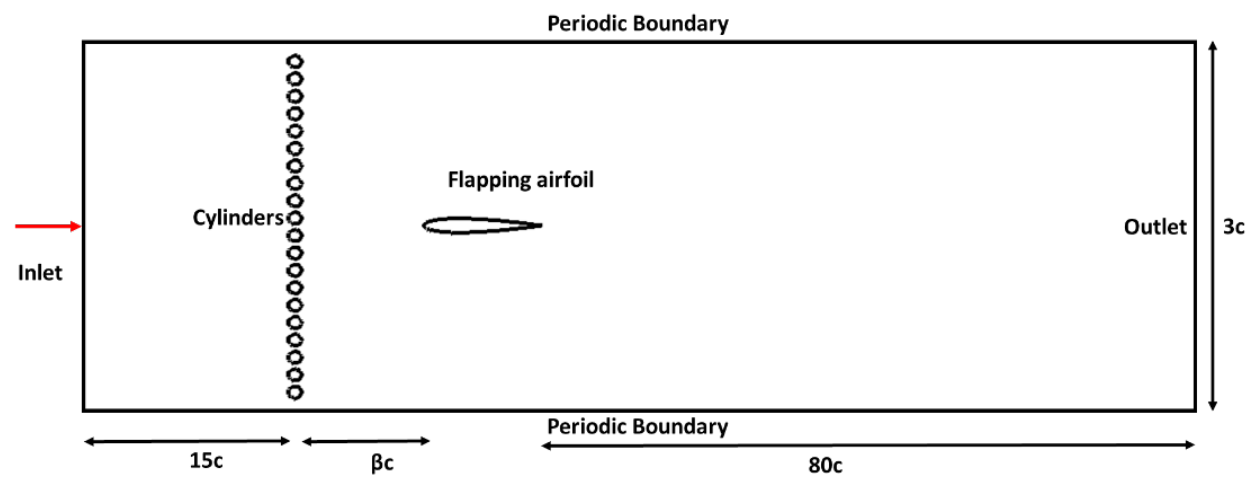

Figure 1. Computational domain of the inline configuration of cylinders with airfoil. 
Placing the arrays of cylinders in an inline or staggered configuration upstream as shown in Figure 1 creates the nonuniform flow conditions. In the same figure, the physical domain and boundary conditions for numerical simulation are illustrated as well. Note that the distance between the cylinder arrays and the airfoil is set as $\beta c$. In this study, $\beta$ varies from 1 to 5 . The meshes near the cylinder arrays and airfoil are presented in Figure 2. The Reynolds numbers $(\mathrm{Re})$ based on the diameter of circular cylinders and chord length of airfoil are 1,000 and 10,000, respectively. The Mach number is fixed at 0.1 for all cases. The airfoil is oscillating sinusoidally around the one-third-chord position $(c / 3)$. The ratio of distance between cylinders $(L)$ to the cylinder diameter $(d)$, i.e. $L / d$, is 1.5 for both configurations. The third order accuracy of space discretization and the time step size of $5 \times 10^{-5}$ are used for the simulations.

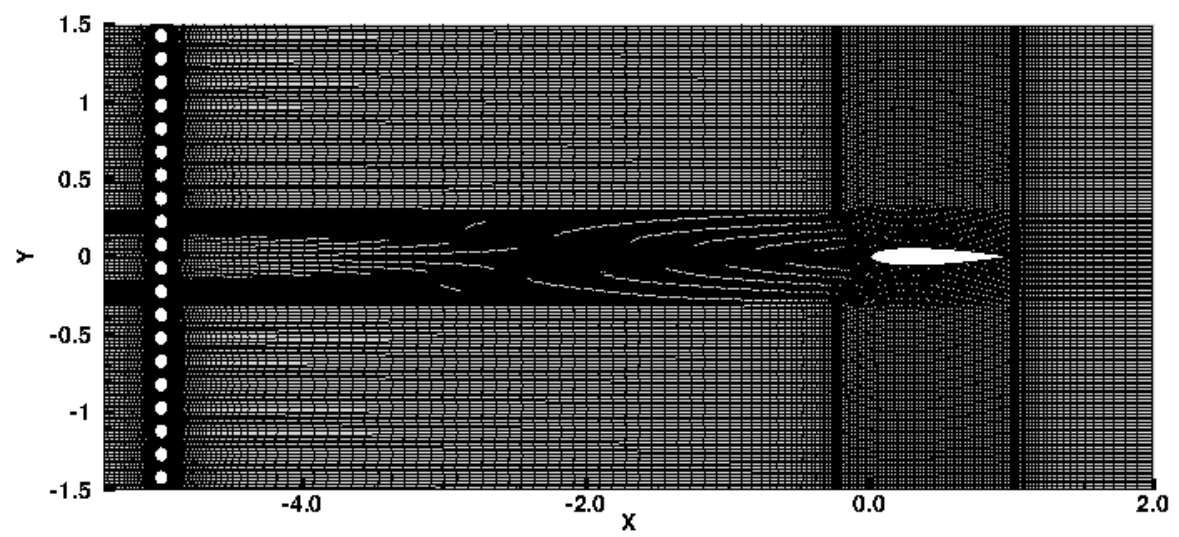

(a)

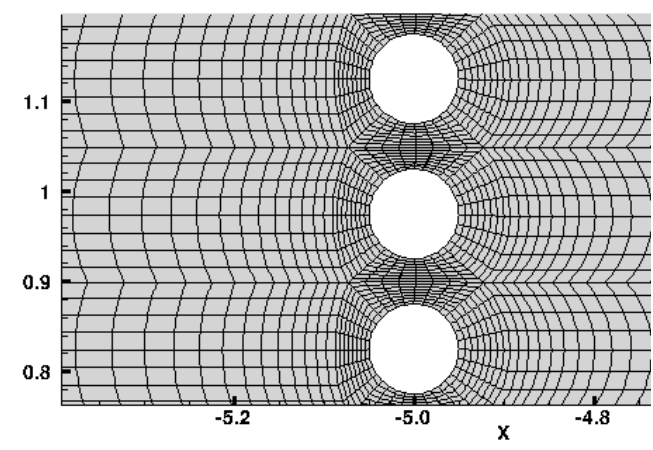

(b)

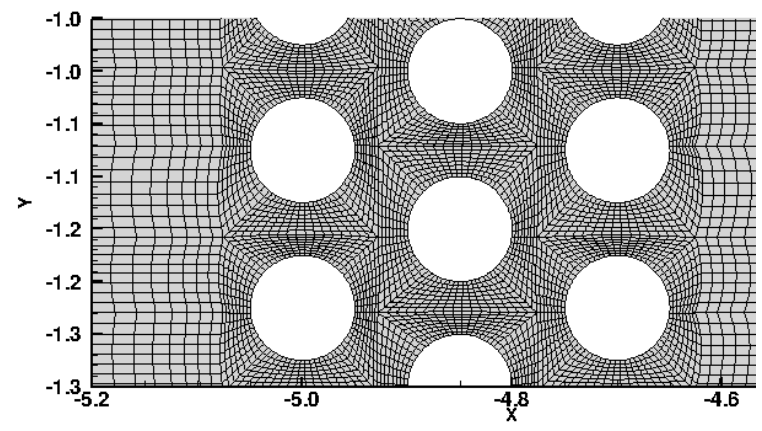

(c)

Figure 2. (a) Computational mesh of the inline configuration of cylinders with airfoil. (b) Close view of meshes for the inline configuration of cylinders. (c) Close view of meshes for the staggered configuration of cylinders.

\section{Results and Discussions}

\section{IV.1. Flow Field Visualization}

Firstly, the flow over the pitching airfoil in the uniform free stream is simulated as a baseline. This is used to compare the flow phenomena with two different configurations of cylinder arrays, namely inline and staggered ones with varying $\beta c$, the distance between array of cylinders and airfoil. Figure 3 shows an instantaneous vorticity field for the pitching airfoil with uniform freestream flow. With the reduced frequency and Strouhal number 4 and 0.3 respectively, the reverse von Karman vortex streets or thrust producing wakes are generated with the vortex row of positive vorticity on the top of the vortex row with negative vorticity causing momentum surfeit. 


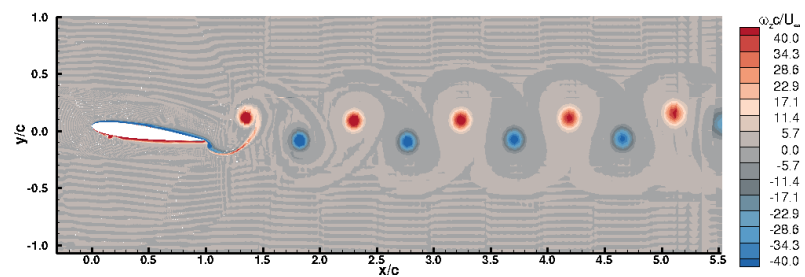

Figure 3. An instantaneous vorticity field at $k=4$ and $S_{t}=0.3$ with uniform freestream flow.

In the Inline configuration, three cases are selected with the distance between array of cylinders and airfoil, $\beta c$ at $1 \mathrm{c}, 3 \mathrm{c}$ and $5 \mathrm{c}$. Figure 4 (a-c) shows the instantaneous vorticity fields for the airfoil pitching in the wakes of inline array of cylinders at $1 \mathrm{c}, 3 \mathrm{c}$ and $5 \mathrm{c}$, respectively. It is clear from Figure 4 that the unsteady ambient flow can affect the formation of leading edge vortices (LEVs) as well as trailing edge vortices (TEVs). The periodic wake structure of the pitching airfoil in a uniform freestream shows chaotic features when interacting with the highly unsteady upstream wakes.

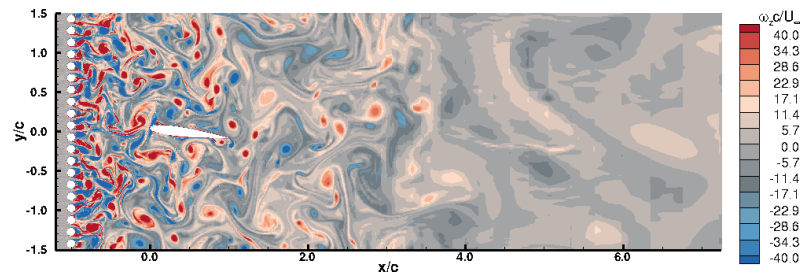

(a)

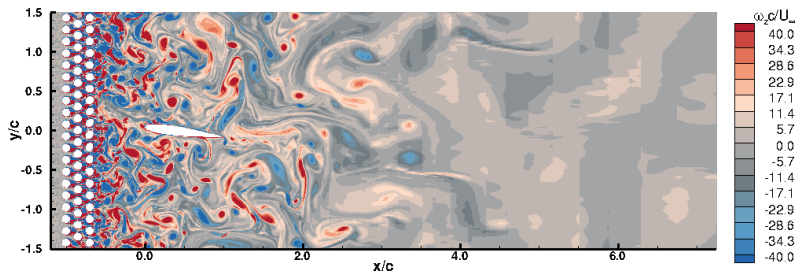

(d)

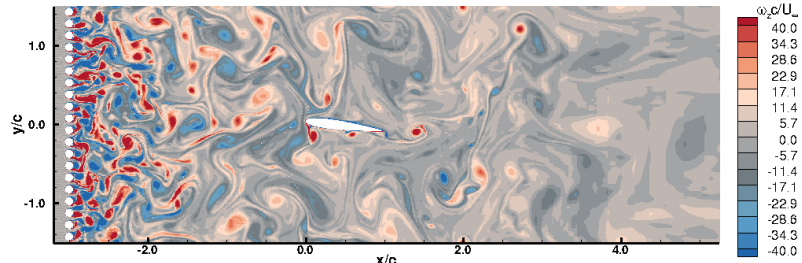

(b)

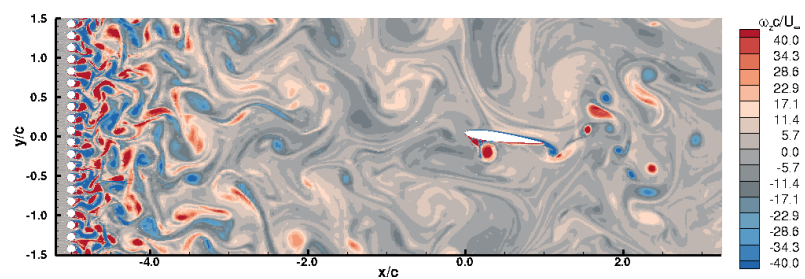

(c)

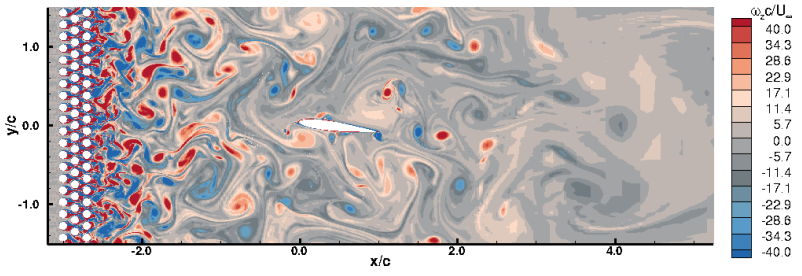

(e)

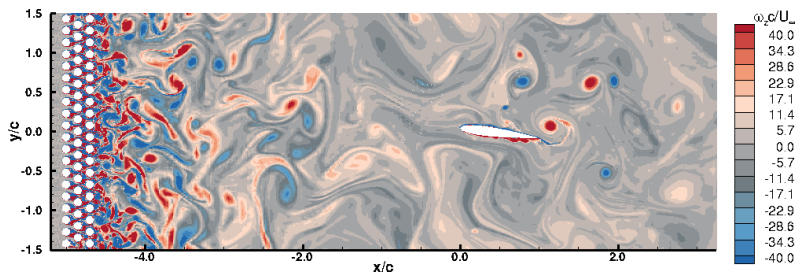

(f)

Figure 4. Instantaneous vorticity fields for $k=4$ and $S_{t}=0.3$ with upstream cylinders in inline and staggered configurations. (a) Inline 1c, (b) Inline 3c, (c) Inline 5c, (d) Staggered 1c, (e) Staggered 3c and (f) Staggered $5 c$.

In the staggered arrangement, three arrays of cylinders are placed in a staggered configuration, and the airfoil is placed at different positions downstream, namely $1 \mathrm{c}, 3 \mathrm{c}$ and $5 \mathrm{c}$. The instantaneous vorticity fields for the staggered cylinder cases after eleven pitching cycles are presented in Figure 4 (d-f). Compared to the unsteady flow environment generated by the inline configuration, the flow under staggered configuration shows stronger flow interference among the cylinders, resulting smaller vortex structures. 


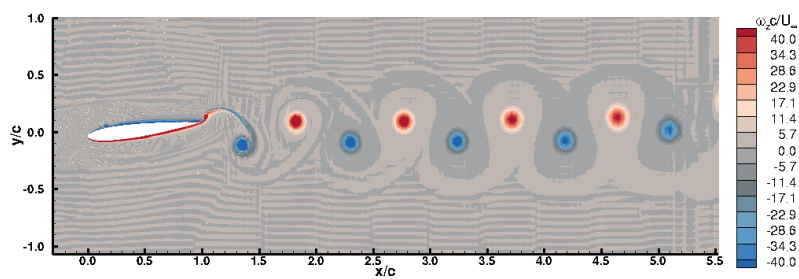

(a)

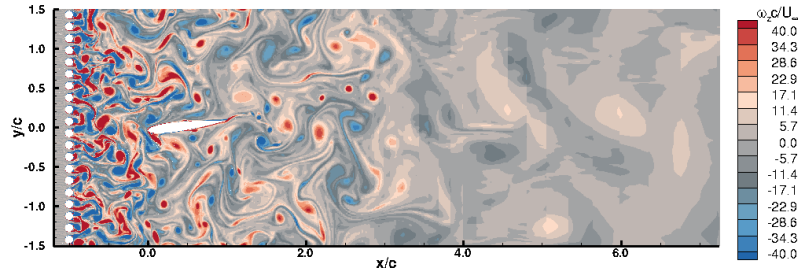

(b)

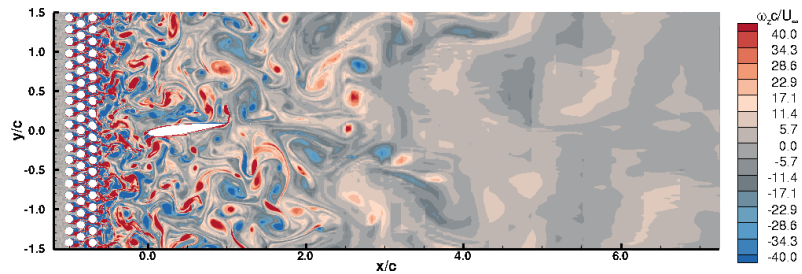

(c)

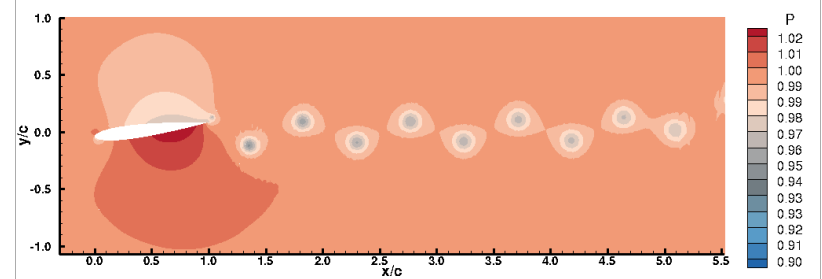

(d)

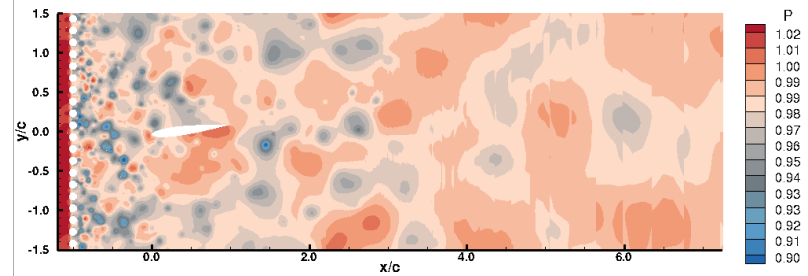

(e)

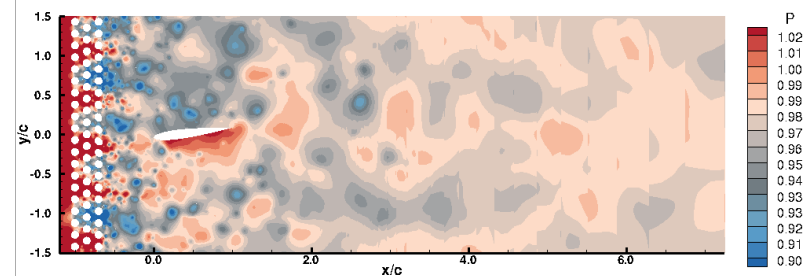

(f)

Figure 5. Instantaneous vorticity and pressure fields for $k=4$ and $S_{t}=0.3$. (a) Vorticity filed with uniform freestream, (b) Vorticity filed for inline 1c, (c) Vorticity field for staggered 1c, (d) Pressure filed with uniform freestream, (e) Pressure filed for inline $1 c$ and (f) Pressure field for staggered $1 c$.

Figure 5 shows the instantaneous vorticity and pressure fields with the staggered and inline upstream cylinders positioned at one chord length along with the baseline case corresponding to the high peak value of the thrust coefficients seen in Figure 6 (a). The positive vortices form the upstream cylinders interact with the pitching airfoil resulting the low pressure and high pressure on upper and lower surface respectively on the airfoil. For the inline configuration the vortices interacting with pitching airfoil are weaker. The pressure fields values for the inline configuration is similar to the baseline case shown in Figure 5 (d-e). It is clear that the Figure 6 (a) the amplitudes of thrust coefficients are similar for inline and baseline cases with small fluctuation.

\section{IV.2. Aerodynamic Forces Analysis}

The time averaged aerodynamic coefficients of thrust $\left(\boldsymbol{C}_{T}\right)$, lift $\left(\boldsymbol{C}_{\boldsymbol{L}}\right)$ and fluctuating lift coefficient $\left(\boldsymbol{C}_{\boldsymbol{T} / L, \boldsymbol{R} M S}\right)$ are defined as:

$$
\begin{gathered}
C_{T}=-\frac{2 F_{x}}{\rho U_{\infty}^{2} A} \\
C_{L}=\frac{2 F_{y}}{\rho U_{\infty}^{2} A} \\
C_{T / L, R M S}=\frac{2 F_{x / y, R M S}}{\rho U_{\infty}^{2} A}
\end{gathered}
$$

where $F_{x}$ is the thrust force, $F_{y}$ is the lift force, $F_{y, R M S}$ is the root mean square of lift force, $\rho$ is the fluid density, $U_{\infty}$ is the freestream velocity and $\mathrm{A}$ is the area. 


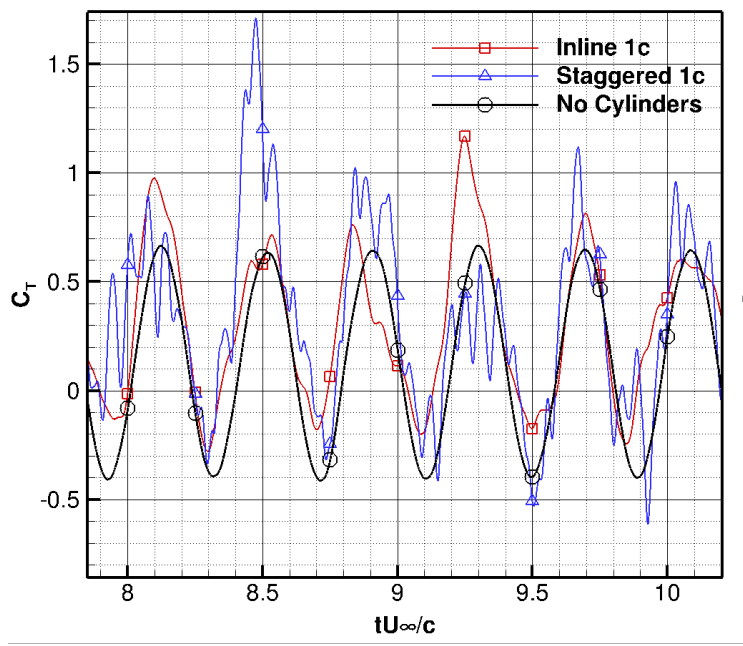

(a)

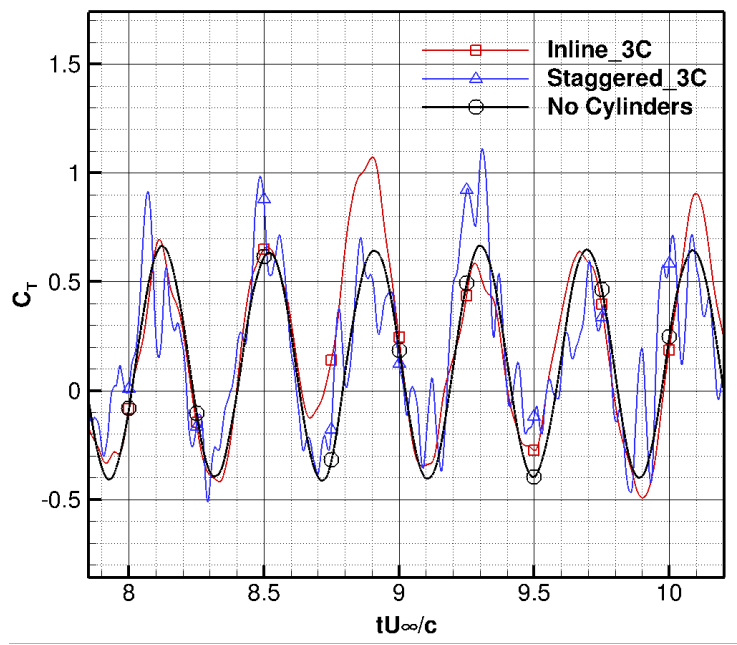

(c)

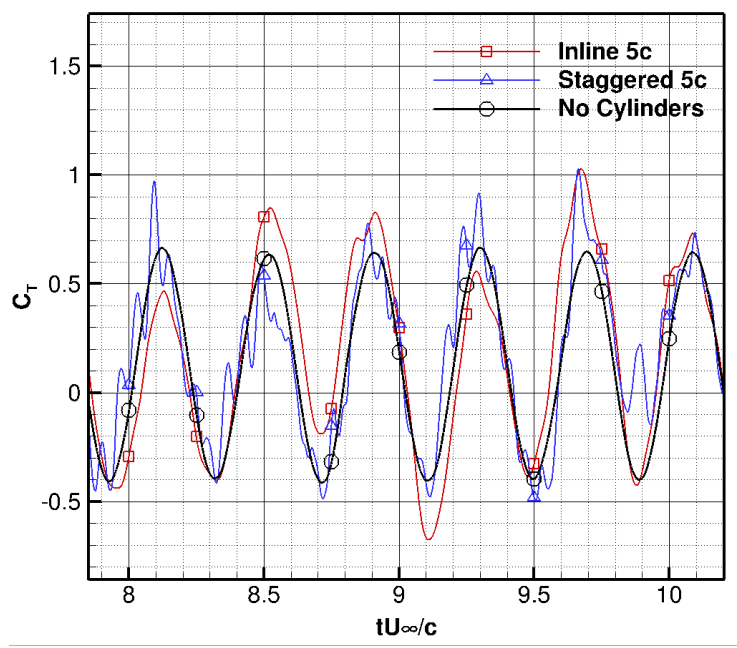

(e)

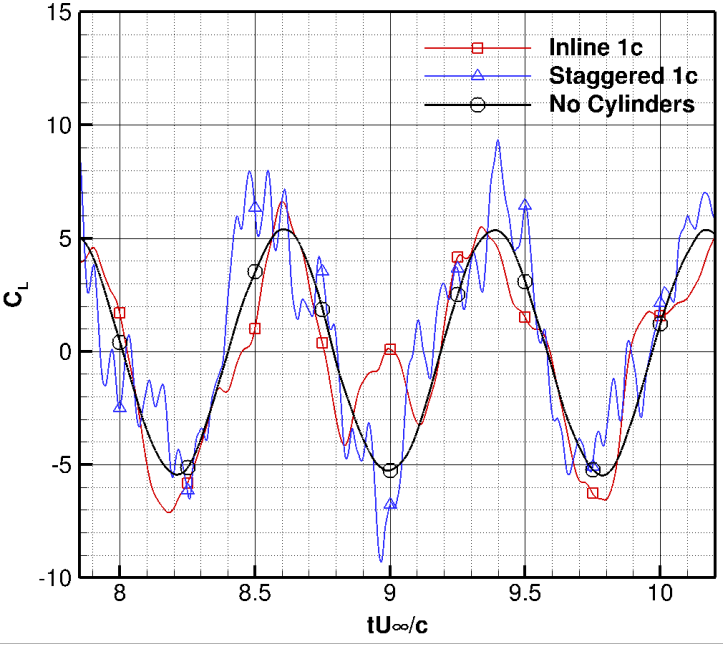

(b)

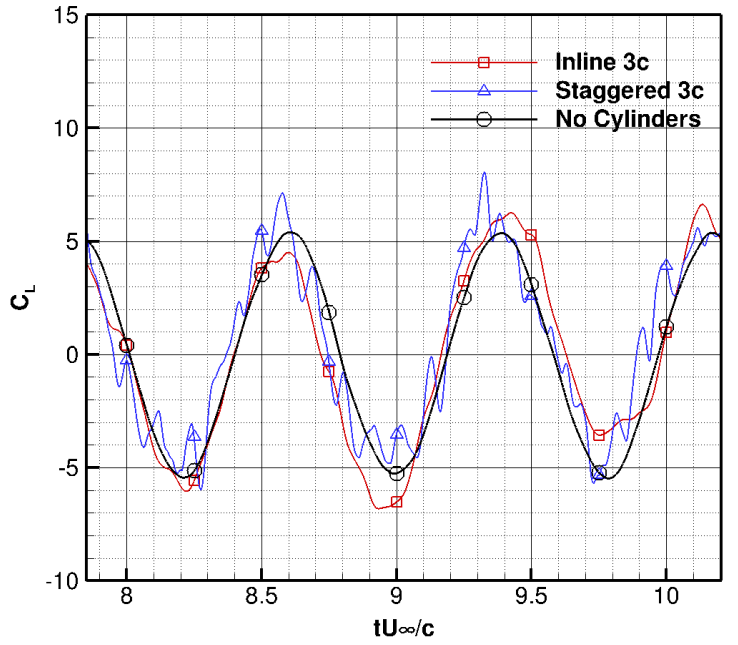

(d)

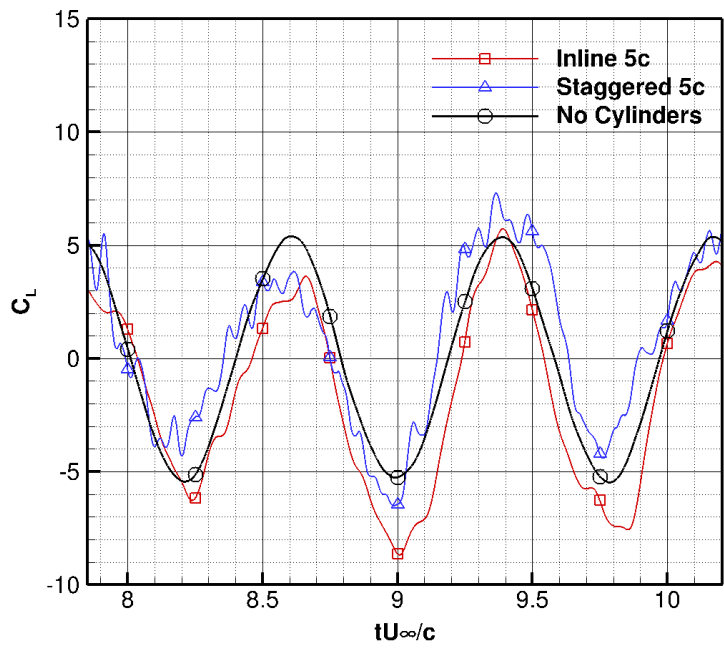

(f)

Figure 6. Thrust and lift coefficients histories for three pitching cycles over airfoil at $k=4$ and $S_{t}=0.3$ (a-b) upstream cylinders configurations at $1 c,(c-d)$ upstream cylinders configurations at $3 c$, and (e-f) upstream cylinders configurations at $5 c$. 
The histories of thrust and lift coefficients for three pitching cycles acting on airfoil are shown in Figure 6. It is observed that the aerodynamic forces acting on the airfoil are quite oscillatory due to the wakes produced by the upstream cylinders. The aerodynamic forces acting on the airfoil are more fluctuating for staggered configurations cases than inline cases due to the vortex structures are more interacting with the pitching airfoil presented in Figure 6. It is obvious from Figure 6 that the peaks of the thrust and lift coefficients are higher for the staggered cases when the airfoil is pitching up.
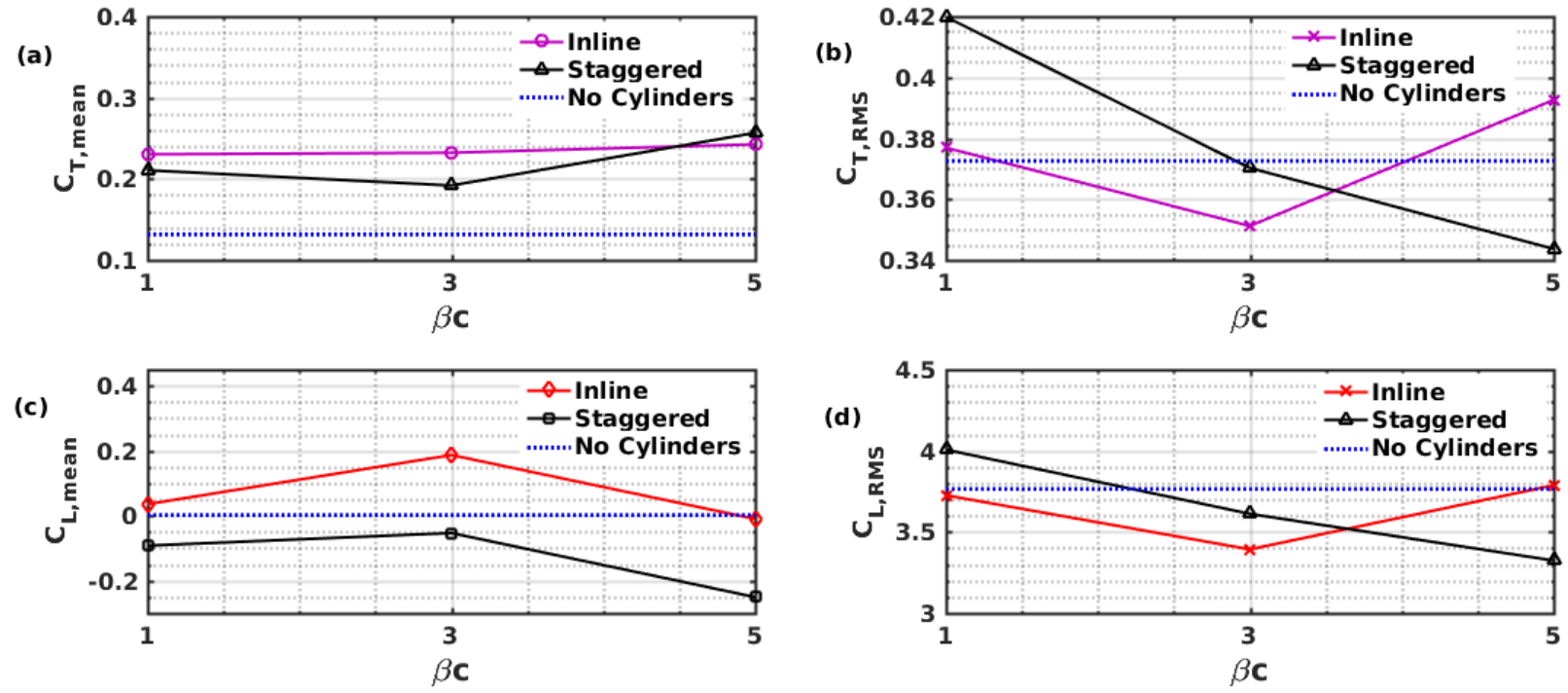

Figure 7. Time averaged aerodynamics forces and their root mean squares.(a) Thrust coefficient, (b) Root Mean Square of thrust coefficient, (c) lift coefficient and (d) Root Mean Square of Lift coefficient at $k=4$ and $S_{t}=0.3$.

Figure 7 shows the time averages of the aerodynamic force coefficients on the airfoil for no cylinders, inline, and staggered configurations at different distances $(\beta c)$ between the arrays of cylinders and airfoil. The time average was performed for last twenty pitching cycles excluding the first five cycles during the simulation for all cases. The thrust coefficient is higher for both inline and staggered configurations compared to the thrust coefficient of airfoil with uniform freestream flow presented in Figure 7 (a). It is observed that the thrust coefficient remains almost the same at various distances between cylinders array and airfoil for the inline configuration. But there is large variation in thrust coefficient values at different distances for staggered configurations. These phenomena are closely related to the interaction between the airfoil and the unsteady environment generated by the cylinder arrays. The underlying mechanisms are still under investigation.

The root mean square (RMS) values of thrust and lift coefficients are shown in Figure 7 (c-d). The RMS values of thrust and lift coefficients are higher for staggered configuration when the distance between the staggered arrays of cylinders positioned at one chord length to airfoil, and decrease as the distance increases to three chord length (3c) and 5 chord length (5c). We note that the RMS values of thrust for the $3 \mathrm{c}$ and $5 \mathrm{c}$ cases are smaller than that of the baseline case without cylinders. However, the mean thrust for these two cases are higher than that of the baseline one. This indicates that the drag is reduced in the $3 \mathrm{c}$ and $5 \mathrm{c}$ cases. The RMS values of the thrust and lift coefficients fluctuate with the distance between the cylinder array and airfoil for the inline configuration. This indicates that there exist relatively large vortices in the wake of the inline cylinder array interacting with the pitching airfoil.

The power spectral density (PSD) analysis of the lift coefficient acting on the airfoil was conducted using periodogram power spectral density function in MATLAB as shown in Figure 8. From the PSD plots presented in Figure 8 (h-n), it is observed that the Strouhal numbers for the cases with no cylinders, inline and staggered configurations are the same. This means that the dominant frequency peak was found 
almost the same as the pitching frequency of the airfoil for every case. In each PSD plots, the amplitude of power is larger for the case of baseline without the cylinders. The peaks for inline and staggered configurations have decreased due to the nonlinear interactions from the wakes of upstream array of cylinders.
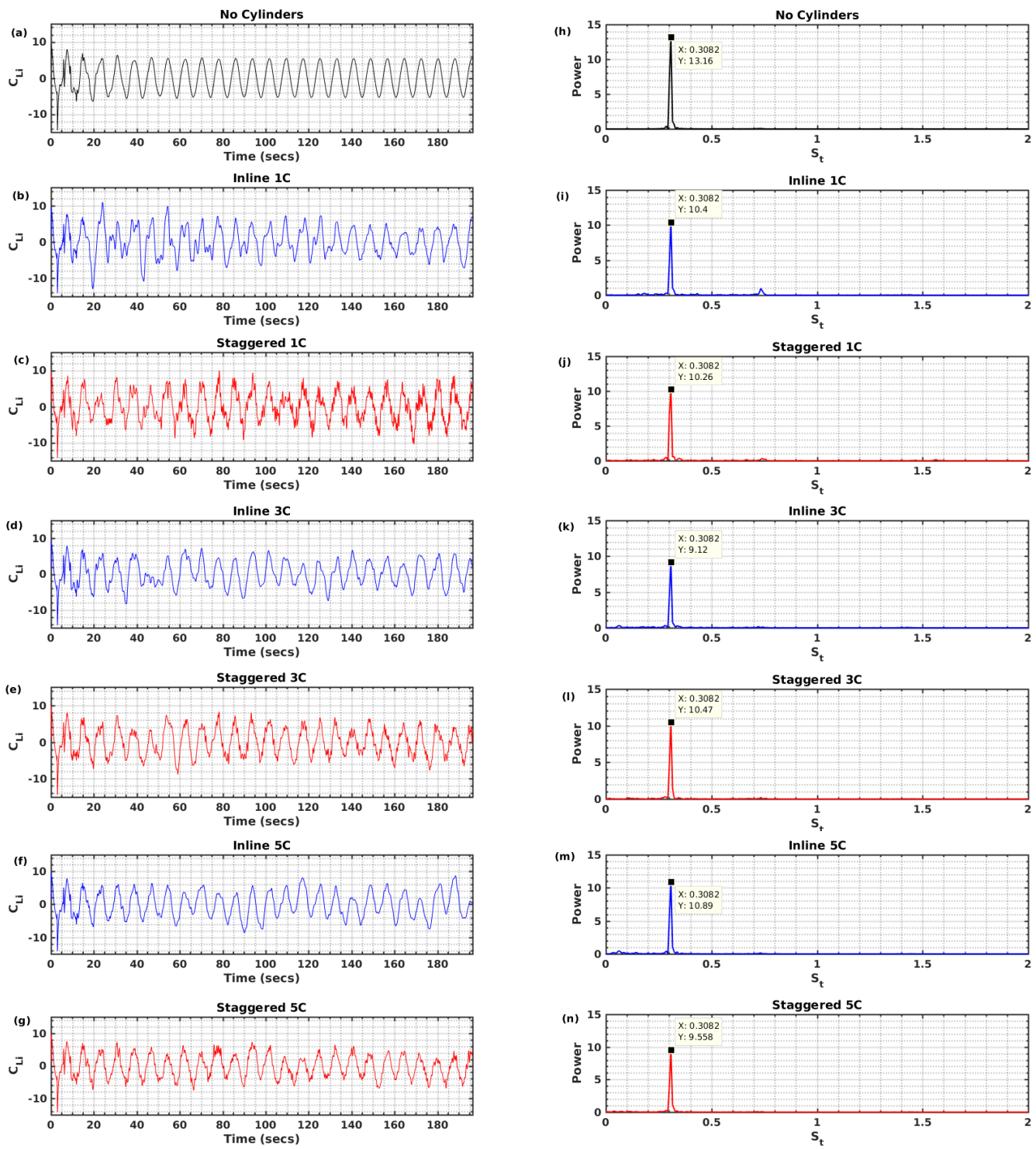

Figure 8. Lift coefficient histories (a) No Cylinders, (b) Inline 1C cylinder configuration, (c) Staggered $1 C$ cylinder configuration, (d) Inline $3 C$ cylinder configuration, (e) Staggered $3 C$ cylinder configuration, (f) Inline 5C cylinder configuration and (g) Staggered 5C cylinder configuration. PSD Plots of Lift Coefficient on airfoil (h) No Cylinders, (i) Inline 1C cylinder configuration, (j) Staggered 1C cylinder configuration, $(k)$ Inline $3 C$ cylinder configuration, (l) Staggered $3 C$ cylinder configuration, (m) Inline $5 C$ cylinder configuration and (n) Staggered $5 C$ cylinder configuration at $k=4$ and $S_{t}=0.3$. 


\section{Conclusion}

The impact of unsteady flow environments on aerodynamic performance of a pitching NACA0012 airfoil is studied in this work. The flow unsteadiness is triggered by the interaction of upstream wakes produced by cylinder arrays placed in two different configurations, namely inline and staggered. The airfoil is located at three different positions, namely one, three and five chord lengths, downstream of the cylinder arrays, and oscillates in the unsteady environment. We observe that the upstream unsteadiness can significantly affect the aerodynamic performance of the flapping wing. A direct flow visualization tells that the unsteady environment can significantly modify the vortex structures around the pitching airfoil, thus affecting aerodynamic forces acting on it. An interesting observation is that in all the tests carried out in this study $\left(\mathrm{Re}=10,000\right.$ based on the airfoil chord length, $\mathrm{k}=4$ and $\left.\mathrm{S}_{\mathrm{t}}=0.3\right)$, the unsteadiness in flow environment can enhance thrust generation. Especially for the staggered configuration, the averaged drag on the pitching airfoil decreases when its distance from the cylinder arrays increases. A PSD analysis is then conducted for the lift coefficients. We observe that the unsteady environments induced by cylinder arrays do no affect the dominant frequency in the flow field.

\section{References}

${ }^{1}$ Ward, T. A., Rezadad, M., Fearday, C. J., and Viyapuri, R., "A Review of Biomimetic Air Vehicle Research: 1984-2014,” International Journal of Micro Air Vehicles, vol. 7, Sep. 2015, pp. 375-394.

${ }^{2}$ Gyllhem, D., Mohseni, K., Lawrence, D., and Geuzaine, P., "Numerical simulation of flow around the Colorado micro aerial vehicle," Jul. 2005, pp. 2005-4757.

${ }^{3}$ Lian, Y., "Numerical Study of a Flapping Airfoil in Gusty Environments," 27th AIAA Applied Aerodynamics Conference, San Antonio, Texas: American Institute of Aeronautics and Astronautics, 2009.

${ }^{4}$ Gopalan, H., "Numerical Modeling of Aerodynamics of Airfoils of Micro Air Vehicles in Gusty Environment," University of Akron, 2008.

${ }^{5}$ Shyy, W., Jenkins, D., and Smith, R., "Study of Adaptive Shape Airfoils at Low Reynolds Number in Oscillatory Flows," AIAA Journal, vol. 35, 1997, pp. 1545-1548.

${ }^{6}$ Wan, T., and Huang, C., "Numerical Simulation of Flapping Wing Aerodynamic Performance under Gust Wind Conditions," 26TH International Congress of The Aeronautical Sciences, 2008.

${ }^{7}$ Prater, R., and Lian, Y., "Aerodynamic Response of Stationary and Flapping wings in Oscillatory Low Reynolds Number Flows," 50th AIAA Aerospace Sciences Meeting including the New Horizons Forum and Aerospace Exposition, American Institute of Aeronautics and Astronautics,

${ }^{8}$ Lian, Y., and Shyy, W., "Laminar-Turbulent Transition of a Low Reynolds Number Rigid or Flexible Airfoil," AIAA Journal, vol. 45, 2007, pp. 1501-1513.

9 Jones, M., and Yamaleev, N., "The Effect of a Gust on the Flapping Wing Performance," 50th AIAA Aerospace Sciences Meeting including the New Horizons Forum and Aerospace Exposition, American Institute of Aeronautics and Astronautics,

${ }^{10}$ Gao, T., and Lu, X. -Y., "Insect normal hovering flight in ground effect," Physics of Fluids, vol. 20, Aug. 2008, p. 087101.

${ }^{11}$ Chen, J. M., and Choa, C. -C., "Freestream Disturbance Effects on an Airfoil Pitching at Constant Rate," Journal of Aircraft, vol. 36, 1999, pp. 507-514.

${ }^{12}$ Wang, S., Zhou, Y., Alam, M. M., and Yang, H. X., "Turbulent Intensity Effect on Low Reynolds Number Airfoil Wake," Fluid-Structure-Sound Interactions and Control, Springer, Berlin, Heidelberg, 2014, pp. 197-202.

${ }^{13}$ Gharali, K., and Johnson, D. A., "Effects of nonuniform incident velocity on a dynamic wind turbine airfoil," Wind Energy, vol. 18, Feb. 2015, pp. 237-251.

${ }^{14}$ Merrill, B. E., and Peet, Y., "Effects of Upstream Disturbances on a Pitching NACA0012 Airfoil," 54th AIAA Aerospace Sciences Meeting, American Institute of Aeronautics and Astronautics. 
${ }^{15}$ FAVIER, D., AGNES, A., BARBI, C., and MARESCA, C., "Combined translation/pitch motion - A new airfoil dynamic stall simulation," Journal of Aircraft, vol. 25, 1988, pp. 805-814.

${ }^{16}$ Kottapalli, S. B. R., "Drag on an oscillating airfoil in a fluctuating free stream," Aug. 1977.

${ }^{17}$ Chirarattananon, P., Chen, Y., Helbling, E. F., Ma, K. Y., Cheng, R., and Wood, R. J., "Dynamics and flight control of a flapping-wing robotic insect in the presence of wind gusts," Interface Focus, vol. 7, Feb. 2017, p. 20160080.

${ }^{18}$ Shi, Z., and Ming, X., "Experimental investigation on a pitching motion delta wing in unsteady free stream," Modern Physics Letters B, vol. 23, Jan. 2009, pp. 409-412.

${ }^{19}$ Favier, D., Belleudy, J., and Maresca, C., "Influence of Coupling Incidence and Velocity Variations on the Airfoil Dynamic Stall," AHS International, 1992.

${ }^{20} \mathrm{Kim}, \mathrm{Y}$., and Xie, Z.-T., "Modelling the effect of freestream turbulence on dynamic stall of wind turbine blades," Computers \& Fluids, vol. 129, Apr. 2016, pp. 53-66.

${ }^{21}$ Jiang, Y., Mao, M.-L., Deng, X.-G., and Liu, H.-Y., "Numerical investigation on body-wake flow interaction over rod-airfoil configuration," Journal of Fluid Mechanics, vol. 779, Sep. 2015, pp. 1-35.

${ }^{22} \mathrm{Yu}, \mathrm{M}$. L., Wang, Z. J., and Hu, H., "A high-order spectral difference method for unstructured dynamic grids," Computers \& Fluids, vol. 48, Sep. 2011, pp. 84-97.

${ }^{23}$ Persson, P.-O., Bonet, J., and Peraire, J., "Discontinuous Galerkin solution of the Navier-Stokes equations on deformable domains," Computer Methods in Applied Mechanics and Engineering, vol. 198, 2009, pp. 1585-1595.

${ }^{24}$ M.-S. Liou, "A sequel to AUSM, Part 2: AUSM+-up for all speeds," J. Comput. Phys., vol. 214, 2006, pp. 137-170.

${ }^{25} \mathrm{~F}$. Bassi, and S. Rebay, "A high-order accurate discontinuous finite element method for the numerical solution of the compressible Navier-Stokes equations," J. Comput. Phys., vol. 131, 1997, pp. 267-279.

${ }^{26} \mathrm{~S}$. Gottlieb, C.-W. Shu, and E. Tadmor, "Strong stability-preserving high-order time discretization methods," SIAM Rev., vol. 43, 2001, pp. 89-112. 